\title{
An echelon-based single shot optical and terahertz Kerr effect spectrometer
}

\author{
Griffin Mead $^{1}$, Ikufumi Katayama ${ }^{2}$, Jun Takeda ${ }^{2}$, Geoffrey A. Blake ${ }^{1,3, a)}$ \\ ${ }^{1}$ Division of Chemistry and Chemical Engineering, California Institute of Technology, Pasadena, \\ California 91125, USA \\ 2Department of Physics, Graduate School of Engineering, Yokohama National University, \\ Yokohama 240-8501, Japan \\ ${ }^{3}$ Division of Geological and Planetary Sciences, California Institute of Technology, Pasadena, \\ California 91125, USA
}

\begin{abstract}
We report on the design and performance of an echelon-based single shot visible/near-infrared spectrometer with adequate sensitivity to measure the nonlinear optical and terahertz Kerr effects in neat molecular liquids at room temperature. Useful molecular information spanning tens of picoseconds can be measured in just a few milliseconds, and the signal-to-noise performance scales favorably with respect to the standard stage scan technique. These results demonstrate the viability of stage-free nonlinear Kerr effect measurements and provide a route for improvements to the speed of future multidimensional Kerr effect studies.
\end{abstract}

\section{INTRODUCTION}

Direct probes of ultrafast molecular dynamics in liquids are of great interest to both experimentalists and theorists seeking to understand fundamental properties of chemical dynamics and energy transfer. Optical and terahertz Kerr effect (OKE/TKE) spectroscopies are particularly useful in this pursuit, offering subpi-cosecond resolution of a liquid's response to a nonlinear perturbation. ${ }^{1-3}$ While easily performed along one time dimension, Kerr effect spectroscopy can be extended to multiple time (frequency) dimensions, revealing fundamental couplings between low energy vibrational and librational modes. ${ }^{4,5}$ One root limitation in the speed of OKE/TKE data acquisition is the need to use motorized delay stages which indirectly sample the molecular response in the time domain. While not a large impediment in one-dimensional studies, scanning multiple delay lines in multidimensional experiments rapidly becomes a limiting factor, dictating some compromise between experimental duration, time (frequency) resolution, and sensitivity.

\footnotetext{
a)Author to whom correspondence should be addressed: gab@gps.caltech.edu. SUPPLEMENTARY MATERIAL

The supplementary material contains further details on the echelon imaging pathway and the data processing code, as well as comparisons of the six solvents' OKE responses measured using stage scan and single shot techniques. Also included are brief discussions on separating the homodyne and heterodyne components of the Kerr effect signals and the quadratic scaling of the signal.
} 
Generally, stage scan limitations have been overcome using specialized gratings, prisms, or spectrally chirped probe pulses. In conjunction with photodiode arrays, these multiplexing techniques can encode many picoseconds of molecular dynamics onto a degree of freedom that can be measured in a single, or a small number of laser shots. A large body of works have described the application of time-to-frequency, time-to-angle, and time-to-space multiplexing techniques to the linear analog of TKE, terahertz time domain spectroscopy (THz-TDS), and to studying irreversible processes and dynamics in optically excited materials. ${ }^{6-13}$ While these techniques are exceedingly useful for probing ultra-fast dynamics of a material's degradation or the profile of a terahertz waveform, we are interested in applying single shot techniques to study Kerr effect phenomena in liquids. Although a few studies have used spectrally chirped probe pulses for single shot OKE measurements of liquids, the application of other single shot techniques is still very much underinvestigated. 14,15

In this paper, we describe the construction of a single shot apparatus based upon a reflective stair step echelon which maps delay time onto the pixel space of a scientific CMOS (sCMOS) camera array. The design provides a 30 ps measurement window which can be recorded at a $1 \mathrm{kHz}$ acquisition rate. This approach eliminates the use of a stage scan and can measure the OKE and TKE responses of simple liquids in as little as $10 \mathrm{~ms}$ (10 laser shots). The echelon technique also avoids fundamental limits in temporal resolution present in time-to-frequency mapping approaches. ${ }^{13}$ We demonstrate good agreement between data recorded using the single shot apparatus and the conventional stage scan approach. We further quantify the signal-to-noise scaling of the single shot approach and find favorable performance relative to using a stage scan. Overall, the performance achieved demonstrates the feasibility of using a reflective stair step echelon to detect nonlinear molecular Kerr effect signals, extending the utility of the reflective echelon technique beyond the well demonstrated linear electro-optic Pockels effect.

\section{EXPERIMENTAL SETUP}

The laser system used in this work was a $1 \mathrm{kHz}$ Coherent Legend UltraShort Pulse (USP) regenerative amplifier seeded with an $80 \mathrm{MHz}$ Coherent Micra oscillator. The Legend output pulses were split, with $85 \%$ of the beam pumping a Light Conversion TOPAS-C travelingwave optical parametric amplifier (OPA) and a small portion of the remainder used for the detection of Kerr effect signals. The output of the OPA was modulated at $500 \mathrm{~Hz}$ with an optical chopper referenced to the $1 \mathrm{kHz}$ regenerative amplifier transistor-transistor logic (TTL) output. Optical Kerr effect (OKE) measurements used an optical pump at $520 \mathrm{~nm}$, produced by mixing the OPA signal beam fundamental at $1500 \mathrm{~nm}$ with $800 \mathrm{~nm}$ light to generate the sum frequency. Residual $800 / 1500 \mathrm{~nm}$ light was removed with two short pass filters. A neutral density filter was used to further attenuate the $70 \mu \mathrm{J}$ optical pump pulse down to $\sim 8 \mu \mathrm{J}$ ( $4 \mathrm{~mW}$ average power at $500 \mathrm{~Hz}$ modulation) such that the beam would not strike a plasma when focused at the sample. (Plasma generation results in spurious peaks scattered throughout the data which cannot be removed through differential chopping and data processing.) After filtering and attenuation, the pump beam was directed through a half wave plate (HWP) to rotate the horizontally polarized pump beam by $45^{\circ}$. The pump beam was then expanded with a $7.5 \times$ magnification off axis parabolic (OAP) mirror telescope and 
focused onto the sample with a third OAP mirror (Fig. 1). The 1/e ${ }^{2}$ radius of the pump spot at the sample was around $60-70 \mu \mathrm{m}$, corresponding to an average power density of 31 $\mathrm{W} / \mathrm{cm}^{2}$ (and an estimated peak power of $>60 \mathrm{GW} / \mathrm{cm}^{2}$ ). A conical hole machined into the rear side of the third OAP mirror provided clearance for the probe beam to pass through with minimal clipping on the sides of the mirror. The exit hole at the OAP front surface was 3 $\mathrm{mm}$ in diameter, which negligibly attenuated the $50 \mathrm{~mm}$ diameter pump beam.

For terahertz Kerr effect (TKE) measurements, dry nitrogen was used to remove water vapor from the terahertz pump path. The unfiltered $500 \mu \mathrm{J}(250 \mathrm{~mW}$ average power at $500 \mathrm{~Hz}$ modulation), $1500 \mathrm{~nm}$ TOPAS signal beam output pumped a $6 \mathrm{~mm}$ diameter $4-\mathrm{N}, \mathrm{N}$ dimethylamino-4'-N'-methyl-stilbazolium tosylate (DAST) organic crystal THz emitter (DAST, Swiss Terahertz). Residual pump light after the DAST emitter was removed with two THz bandpass filters (QMC Instruments). The THz pump followed the same path as the optical pump, with the addition of a wire grid polarizer (WGP) in the collimated region between OAP 2 and OAP 3 to enforce the $45^{\circ}$ pump polarization. The THz pump pulse energy was $\sim 1.5 \mu \mathrm{J}$ and was focused by the third OAP to a $1 / \mathrm{e}^{2}$ radius of $200 \mu \mathrm{m}$ at the sample, producing an average power density of $0.6 \mathrm{~W} / \mathrm{cm}^{2}$ (and an estimated peak power exceeding $1 \mathrm{GW} / \mathrm{cm}^{2}$ ).

Adapting previously reported designs, the probe beam passed through two reflective telescopes pairs, of which each provided a $8 \times$ magnification of the probe beam. ${ }^{16-18}$ An iris was also placed between the two telescope pairs to further improve the final beam profile homogeneity. The probe beam diameter after magnification is $50 \mathrm{~mm}$, and so completely illuminates the 1000 step nickel echelon (step width $=35 \mu \mathrm{m}$; step depth $=5 \mu \mathrm{m}$ ). This echelon geometry provides $30 \mathrm{ps}$ of total optical delay, with $30 \mathrm{fs}$ of delay between adjacent "beamlets." The reflected probe beam was then directed off a $50 \mathrm{~mm}$ diameter gold mirror before being focused with a Barlow lens combination $(\mathrm{L} 1, \mathrm{~L} 2)$ through the hole in the third OAP. Immediately before passing through the OAP, the probe beam polarization was conditioned with a 10 000:1 preparatory polarizer (P1) and a quarter wave plate (QWP). The energy of the probe beam at this position was $600 \mathrm{~nJ}$, and focused into a $1 / \mathrm{e}^{2}$ radius spot of $73 \mu \mathrm{m}$. This corresponds to an average power density of $3.56 \mathrm{~W} / \mathrm{cm}^{2}$, which is several fold larger than the $\mathrm{THz}$ average power density. However, the probe power is not contained in a few hundred femtosecond pulse but across $30 \mathrm{ps}$, which drops the instantaneous power density by several hundredfold (to on the order of $100 \mathrm{MW} / \mathrm{cm}^{2}$ such that the THz pump power density is much greater than the probe power density).

The orientation of the probe and pump polarizations are aligned such that the measured OKE/TKE signals arise from the anisotropic component of the molecular response function $\left(R_{\text {aniso }} \propto R_{X Y X Y}\right) \cdot{ }^{19}$ A traditional optical heterodyne detection scheme was employed to improve the strength of the Kerr effect signals. ${ }^{20}$ The fast axis of the QWP was aligned parallel to the P1 polarizer axis, and then the polarizer was slightly detuned $\left(\sim 2^{\circ}\right)$ to introduce a small amount of orthogonally polarized light, which acquires from the QWP a $\pi / 2$ phase shift relative to the majority of the probe beam. This small quadrature component acts as a local oscillator during the heterodyne detection of the rotated probe light. When the local oscillator and signal fields arrive at the camera array, each square law detector (camera pixel) produces a signal proportional to the square of the two incident fields 


$$
\left(E_{L O}+E_{s i g}\right)^{2}=E_{L O}^{2}+E_{s i g}^{2}+2 E_{L O} E_{s i g} .
$$

This equation is a valid approximation for the single shot measurements, where both the preparatory polarizer detuning angle and induced birefringence in the sample are small. The polarizer angle can be adjusted to ensure that the local oscillator background is always much greater than the Kerr effect response, which ensures the signal is dominated by the term in Eq. (1) that is linearly proportional to $\mathrm{E}_{\text {sig. }}$. The ultimate limit to the intensity of the local oscillator background is the well depth of the camera pixels. The probe intensity in these experiments corresponded to $\approx 3000$ total photon counts per individual unbinned pixel per image (or 10000 incident photons at a quantum efficiency (QE) of $30 \%$ at $800 \mathrm{~nm}$ ). This probe illumination intensity falls well within the linear photon counting regime of the camera, which has a linearity greater than $99 \%$ of the well depth of $30000 e^{-}$.

In OKE measurements, a $1 \mathrm{~mm}$ path length Suprasil QS cuvette contained the sample, while for TKE measurements, a $5 \times 5 \mathrm{~mm}^{2}$ clear aperture, $1 \mu \mathrm{m}$ thick silicon nitride window on a $10 \times 10 \mathrm{~mm}^{2} \mathrm{Si}$ substrate (Norcada) was epoxied in place over a glass cuvette with a $6.5 \mathrm{~mm}$ diameter hole drilled through one wall, creating an effective path length of $1.5 \mathrm{~mm}$. After interacting with the pumped sample, the probe beam passed through a second 10 000:1 analyzing polarizer (P2) which was crossed at $90^{\circ}$ with respect to the fast axis of the QWP. The probe beam then passes through two cylindrical imaging lenses (L3, L4) and a $750 \mathrm{~nm}$ long pass filter (LPF) before hitting the camera's sCMOS array. Proper imaging of the echelon surface onto the camera array is absolutely critical for ensuring good quality measurements. The imaging pathway, as well as specific properties and positions of the postechelon Barlow and imaging lenses are presented in the supplementary material.

Data were acquired at $1 \mathrm{kHz}$ using a 10-tap Andor Zyla 5.5 MP camera and the Andor Solis program. Acquisitions were triggered from the regenerative amplifier delay generator, and the exposure time was set to $800 \mu$ s so that only a single laser pulse was captured in each image. Chopping the pump beam at $500 \mathrm{~Hz}$ allowed for data to be acquired in an on-off manner, which compensated for drift from shot-to-shot fluctuations in beam intensity and pointing. Each data set consisted of 10000 images (5000 on, 5000 off) that were acquired in $10 \mathrm{~s}$ and saved to disk in ".dat" format. A Python script was then used for data processing, and it is included in the supplementary material.

After reshaping the raw data to match the camera dimensions and the number of images acquired, odd and even numbered images were separately coadded. The two data sets (corresponding to pump on and pump off conditions) were then subtracted and normalized by the pump off data set [Eq. (2)],

$$
S i g_{\text {O/TKE }}=\frac{S i g_{O N}-S i g_{O F F}}{S i g_{\text {OFF }}} .
$$


As the pump off data set corresponds to $E_{L O}^{2}$ the subtraction of the two data sets removes this term. In the experimental case of $E_{L O}$ » $E_{s i g}$, the resulting signal is now $E_{\text {sig }}^{2}+2 E_{L O} E_{\text {sig }} \approx 2 E_{L O} E_{\text {sig }}$.

For traditional O/TKE studies using single element photodiodes, the small nonlinear homodyne component can be removed in a few different ways; for example, through performing two measurements with the preparatory polarizer oriented at $\pm \phi$, or through measuring the difference signal recorded by a pair of photodiodes after passing through the sample and a series of postsample polarization optics. By contrast, the simultaneous measurement of $E_{L O}^{2}$ in the single shot experiment allows $E_{s i g}$ to be numerically calculated using a single data set. Once determined, the heterodyne and homodyne components can be easily separated. For data throughout this study, the very small homodyne component was not removed from the stage scan or echelon data. Instead, we demonstrate the deconstruction of the echelon data into its homodyne and heterodyne components, thus verifying the necessary condition $E_{L O} \gg E_{\text {sig }}$ (see the supplementary material).

To improve sensitivity, a 2 pixel horizontal bin and an 8 pixel vertical bin were applied to the $2560 \times 80$ pixel subarray of the camera used to acquire data. The 8 pixel vertical binning was aligned parallel to the long axis of the beamlets, and so binning in this direction had no impact on the temporal resolution of the experiment. Coadding along the vertical dimension produced a final data array of $1280 \times 1$ values. Accounting for small regions on each side of the camera array which were not illuminated by the probe beam, the 1000 echelon steps were imaged onto an area that horizontally spanned around $11002 \times$ binned pixels (or 2200 total pixels). This slight oversampling of the probe beamlets ensured that the temporal dynamics encoded by the probe were fully resolved by the camera.

Calibrating the pixel-to-time mapping was achieved by translating a delay stage on the probe beam path by $1.4989 \mathrm{~mm}$ (10 ps of delay), which resulted in the echelon signal peak shifting along the array by $z$ pixels. (A similar calibration could be easily achieved without a delay line by using a small plate of material with a known thickness and index at $800 \mathrm{~nm}$.) A cross correlation between the $\mathrm{t}=0 \mathrm{ps}$ and $\mathrm{t}=10 \mathrm{ps}$ data sets calibrated the time axis, and the time resolution per pixel was found to be $\sim 28$ fs. This provided a Nyquist-limited bandwidth of $17 \mathrm{THz}$. Unlike the stage scan technique whose Nyquist-limited bandwidth is easily tuned by changing the sampling rate and speed of the stage scan, the echelon measurements are less flexible due to the finite number of beamlets and detector elements.

While the Nyquist-limited sampling bandwidth set by the echelon imaging onto the sCMOS array is $17 \mathrm{THz}$, the group velocity dispersion introduced by optics along the probe beam path prior to the sample could reduce the bandwidth by broadening the probe pulse duration. To combat dispersion, the probe beam (initially 51.9 fs after being split from the main Legend beam) makes 6 reflections off of a pair of negative group-velocity dispersion (GVD) mirrors immediately prior to the reflective telescope. This precompensation effectively negates the dispersion introduced by two focusing lenses, the polarizer, the quarter wave plate, and the sample cuvette. The final probe pulse duration immediately before the sample was estimated to be $56.8 \mathrm{fs}$, which sets the upper practical bandwidth at $\sim 7.7 \mathrm{THz}$. 


\section{EXPERIMENTS AND DISCUSSION}

\section{A. Kerr effect measurements}

The temporal resolution of the system was evaluated using dimethyl sulfoxide (DMSO), which has a OKE signal dominated by an instantaneous electronic response that follows the square of the optical pump electric field. The stage scan and echelon methods were both used to measure the response of DMSO and produced nearly identical results [Fig. 2(a)].

The full-width at half maximum of the DMSO response measured using the stage scan was $283.8 \mathrm{fs}$, and that for the echelon data was $285.5 \mathrm{fs}$. As demonstrated by the difference between the stage scan and echelon data (Diff, offset -0.2), good agreement between the two techniques is achieved, and this confirms that the echelon is imaged properly onto the sCMOS array. Transforming the DMSO data into the frequency domain reveals the echelon data has a signal-to-noise transition around $7.7 \mathrm{THz}$, with similar results from the stage scan data [Fig. 2(b)].

The small oscillations apparent in the difference between the DMSO data prompted further investigation. To completely remove any molecular orientational response, the empty Suprasil quartz cuvette was directly measured using the two techniques by shifting the focal region from the cuvette volume to the cuvette wall [Fig 2(c)]. While the instantaneous OKE response is similar between the two techniques (FWHM echelon $=299.5 \mathrm{fs}$, FWHM stage $=$ $280.9 \mathrm{fs}$ ), diffraction patterns in the echelon data are visible. The diffraction signal is visible only in the echelon data because the camera provides far greater spatial resolution than a single photodiode. The sinc-like shape of the diffraction pattern is attributed to the probe beam passing through the $3 \mathrm{~mm}$ circular aperture in the third OAP. While such diffraction occurs with radial symmetry in the direction of probe propagation, binning and coadding along the vertical axis results in only diffraction along the horizontal axis of the camera array being resolved.

The aperture diameter responsible for the diffraction artifacts can be calculated using the pixel length, the distance from the OAP surface to camera array, and the magnification factors contributed by the lenses. Applying the standard relationship $\theta_{0}=1.22 \lambda / \mathrm{D}$ between the photon wavelength, $\lambda$, and the angle formed between the diffraction maxima and first minima, $\theta_{0}$, returns an estimated aperture diameter of $7.2 \mathrm{~mm}$. This is in reasonable agreement with the $3 \mathrm{~mm}$ diameter opening in the OAP, especially given that errors can be introduced from estimating the very small angle $\theta_{0}$ (which is only on the order of ten thousandths of a radian).

While diffraction from the OAP aperture is always present in every image acquired by the camera, probe photons rotated by the nonlinear perturbation in the sample allows an excess number of diffracted photons to accumulate in the pump-on image subset, which subsequently cannot be removed during data processing. Fortunately, these artifacts have a known functional form, and so are amenable to removal by deconvolution techniques. Minimization of the probe beam diameter prior to passage through the OAP aperture would further mitigate these diffraction effects. 
Diffraction from passage of the probe beam through the nonlinear aperture created by the pump field in the sample was also considered. However, the pump electric field cross section is Gaussian, and thus, should produce a nonlinearly perturbed region in the sample that also follows a Gaussian distribution. This Gaussian aperture would subsequently yield a Gaussian diffraction pattern which would not produce the oscillatory side-lobes observed in the data. This qualitative observation, coupled with the good agreement between actual and calculated aperture diameters, supports the OAP hole as the source of the weak diffraction artifacts.

Next, the single shot apparatus was used to measure the OKE and TKE responses of carbon disulfide $\left(\mathrm{CS}^{2}\right)$. For the single shot experiments, 10000 images spanning $30 \mathrm{ps}$ of delay were acquired in $10 \mathrm{~s}$, while the stage scan required $\sim 18 \mathrm{~s} \mathrm{(18} 000$ shots) at $250 \mu \mathrm{m} / \mathrm{s}$ to acquire the same $30 \mathrm{ps}$ of data. The third order nonlinear constant of $\mathrm{CS}_{2}$ is much larger than DMSO $\left(\mathrm{CS}_{2} \operatorname{Re} \chi^{3}=93.17 \times 10^{-24} \mathrm{~m}^{2} / \mathrm{V}^{2} \mathrm{cf}\right.$. DMSO Re $\left.\chi^{3}=14.22 \times 10^{-24} \mathrm{~m}^{2} / \mathrm{V}^{2}\right)$, and the Kerr effect signals are characterized by a slowly decaying molecular orientational response that extends for many picoseconds after the instantaneous electronic response maximum. ${ }^{22,23}$ As seen in Figs. 3(a) and 3(b), the OKE and TKE responses of $\mathrm{CS}_{2}$ are captured with good fidelity using both techniques. Differences in the optical and terahertz Kerr effect responses arise from different contributions from the polarizability and dipole moment operators in the third order response function. ${ }^{5}$ The good agreement between techniques is especially promising when the difference in instantaneous probe photon flux between the two methods is considered. While the entire photon flux is contained within the probe pulse duration using the stage scan method, the echelon disperses the same total number of photons over a $30 \mathrm{ps}$ window, reducing the instantaneous photon flux interacting with the sample by roughly two orders of magnitude.

To examine the quality of the single shot signal as a function of the number of averages, we extracted from the $\mathrm{CS}_{2} \mathrm{~N}=10000$ shot data subsets ranging from $\mathrm{N}=5000$ to $\mathrm{N}=10$ shots. In Figs. 3(c) and 1(d), the Kerr effect responses of $\mathrm{CS}_{2}$ across 4 orders of magnitude of sampling are shown. After 10 shots are acquired the decaying OKE and TKE responses out to several picoseconds are already clearly present. Further sampling extends the decaying response in time and reduces noise. In contrast to the $30 \mathrm{ps}$ of data acquired by the echelon, a stage scan measurement at an equivalent Nyquist-limited bandwidth of $17 \mathrm{THz}$ could only collect around a quarter picosecond of data in $10 \mathrm{~ms}$ (optimistically assuming no limitations are imposed by the mechanics of the delay stage).

Finally, we measured the OKE response of bromoform $\left(\mathrm{CHBr}_{3}\right)$ with the single shot apparatus. Bromoform is a halogenated methane with two low frequency vibrational modes. The full OKE response of bromoform is seen in Fig. 4(a), with the inset highlighting the oscillatory molecular coherences in the data. After detrending a double exponential decay from the data [Fig. 4(a) inset, dashed red line], the residual was Fourier transformed, with two strong features confirming the presence of the molecular modes at $4.66 \mathrm{THz}$ ( $V_{6}$, lit. $4.64 \mathrm{THz})$ and $6.73 \mathrm{THz}\left(v_{3}\right.$, lit. $\left.6.68 \mathrm{THz}\right)$, the later of which is at the upper bounds of the practical experimental bandwidth. With the optical pump non-resonant with these two modes, only a nonlinear two-photon Raman process can be responsible for the detection of these features. Similar molecular coherences were observed in diiodomethane (CH2I2), with 
the $3.65 \mathrm{THz}\left(v_{4}\right.$, lit. $\left.3.65 \mathrm{THz}\right)$ mode clearly visible. ${ }^{25}$ The diiodomethane data may be found in the supplementary material. Dichloromethane, which has a Raman active mode at 8.5 THz was also measured but no coherences were observed, a finding consistent with the bandwidth limitations imposed by the probe beam duration.

\section{B. Noise performance and sensitivity}

The signal-to-noise characteristics of the echelon technique were quantified by calculating the root-mean-square (rms) noise in the OKE response of a series of solvents, and comparing these values to that of stage scan data. The solvents were chosen to span a broad range of $\chi^{3}$ values, which are directly related to the magnitude of the Kerr response of the liquid. The six liquids measured were acetonitrile $\left(\operatorname{Re} \chi^{3}=6.61 \mathrm{pm}^{2} / \mathrm{V}^{2}\right)$, acetone $\left(\operatorname{Re} \chi^{3}=\right.$ $\left.10.46 \mathrm{pm}^{2} / \mathrm{V}^{2}\right)$, dimethylsulfoxide $\left(\operatorname{Re} \chi^{3}=14.22 \mathrm{pm}^{2} / \mathrm{V}^{2}\right)$, nitroben-zene $\left(\operatorname{Re} \chi^{3}=21.02\right.$ $\left.\mathrm{pm}^{2} / \mathrm{V}^{2}\right)$, benzene $\left(\operatorname{Re} \chi^{3}=34.34 \mathrm{pm}^{2} / \mathrm{V}^{2}\right)$, and carbon disulfide $\left(\operatorname{Re} \chi^{3}=93.17 \mathrm{pm}^{2} / \mathrm{V}^{2}\right) .{ }^{22}$

For each liquid, the mean-corrected percent rms $(\% \sigma)$ of a 3 ps region of the data before the molecular signal was calculated and normalized to the peak of the molecular signal.

Comparison data were also acquired using the stage scan method. To keep the information content of the two techniques consistent, the sampling rate of the data acquisition card used for stage scan measurements was adjusted such that the Nyquist-limited bandwidth was 17 THz. All stage scans collected 30 ps of data, while the stage scan velocity was adjusted to change the number of laser shots acquired in the data sets. The full data sets for each solvent, including comparisons between echelon and stage scan data, as well as extraction of the heterodyne and homodyne components of the echelon data, are included in the supplementary material.

Across the range of weakly to strongly OKE-active liquids, the echelon data had a linear, 1/ W relationship between $\log (N)$ and $\log (\% \sigma)$. The rms performance for OKE measurements of acetonitrile and carbon disulfide are shown in Fig. 5(a). The 1/ $\mathbb{W}$ scaling was constant across 4 orders of sampling magnitude, as demonstrated by the line of best fit. Thus, random Gaussian noise appears to be the predominant noise source in the echelon data. A weaker relationship between the number of shots and the stage scan $\% \sigma$ was found, indicating the data were limited by correlated noise in the measurement and were approaching the noise floor of the stage scan technique for the given experimental parameters.

Next, the rms performance of the $\mathrm{CS}_{2}$ TKE response was measured using both the echelon and stage scan techniques. The rms behaviors of the two techniques were equivalent in the OKE and TKE experiments, although the TKE response was overall weaker, requiring more measurements to achieve the same rms noise [Fig. 5(b)]. A major cause of the weaker TKE response was the lower terahertz pump energy. For example, at $\mathrm{N}=10000$ shots, the measured difference in $\log (\% \sigma)$ between TKE and OKE echelon measurements was 0.88 . Given that the Kerr effect signal scales linearly with the pump power (Watts $\propto I=\left|E^{2}\right|$ ), a difference in $\log (\% \sigma)$ of 1.7 was calculated based upon the ratio of the optical and terahertz pump energies $\left[1.7=\log \left(31 \mathrm{~W} / \mathrm{cm}^{2} / 0.6 \mathrm{~W} / \mathrm{cm}^{2}\right)\right]$, which agrees well with experiment considering the difficulty in measuring the $\mathrm{THz}$ pump power with the same precision as the OKE pump power. 
A clear trend was observed in the performance of the two techniques across the range of solvents measured with OKE. In Fig. 5(c), the $\log (\% \sigma)$ after accumulating 10000 shots was plotted against the logarithm of the third order nonlinear susceptibility $\chi^{3}$ of each solvent. While solvents with weaker OKE responses tend to reach similar noise floors after 10000 shots, irrespective of the measurement technique used, the echelon measurements consistently reach lower rms values for even moderately OKE active liquids.

Finally, the sensitivity of the echelon technique was also investigated. For each solvent measured, the total number of signal photons (given by the difference between pump on and pump off data sets) was normalized by the total number of photons in the pump off data set. An identical analysis was performed using a data set with the pump beam blocked, which measures the random error in background photon subtraction for a given probe intensity, and provides a measure of the absolute noise floor of the experiment. As shown in Fig. 5(d), even the weakest OKE response corresponding to a modulation on the order of $0.1 \%$ was orders of magnitude larger than the absolute experimental noise floor, which was around $0.001 \%$.

\section{CONCLUSIONS}

A single shot, reflective echelon spectrometer design has been shown to acquire accurate OKE and TKE data of simple liquids and has sufficient sensitivity to record tens of picoseconds of molecular signals in as few as 10 laser shots $(10 \mathrm{~ms})$. Furthermore, the detection of Raman-active molecular coherences in simple halo-genated methanes is especially promising for future applications of the echelon technique to multidimensional nonlinear spectro-scopies. Finally, the noise performance of the echelon approach is found to be very competitive to the standard stage scan technique. In sum, these results highlight the feasibility of stage-free nonlinear spectroscopic measurements with orders-of-magnitude faster acquisition times.

As the technique is developed further, a series of questions remain to be explored. In particular, how strongly does the data quality depend upon the camera frame rate and pixel linearity? If data are found to be relatively robust to these parameters, then the methodology outlined above could perform well with a broader set of commercially available cameras, providing a reasonable alternative to motorized stages for performing high resolution nonlinear spectroscopy.

\section{Supplementary Material}

Refer to Web version on PubMed Central for supplementary material.

\section{ACKNOWLEDGMENTS}

This research is based upon work supported by the National Science Foundation Graduate Research Fellowship under Grant No. DGE 1745301. This research was further supported by grants from the National Science Foundation Chemical Structure, Dynamics, and Mechanisms program (Grant No. CHE-1665467), from the National Aeronautics and Space Administration Astrophysics Research and Analysis (Grant No. NNX16AC75G) and Astrobiology (Grant No. NNX15AT33A) programs, and from the Grants-in-Aid for Scientific Research (Grant Nos. 18H04288, 16H04001, and 17H06124) from the Ministry of Sports, Culture, Science, and Technology, Japan. 


\section{REFERENCES}

1. Bartolini P, Taschin A, Eramo R, and Torre R, "Optical Kerr effect experiments on complex liqiuds," in Time-Resolved Spectroscopy in Complex Liquids: An Experimental Perspective (Springer, 2008), pp. 73-127.

2. Hoffmann MC, Brandt NC, Hwang HY, Yeh KL, and Nelson KA, “Terahertz Kerr effect," Appl. Phys. Lett 95,231105 (2009).

3. Allodi MA, Finneran IA, and Blake GA, "Nonlinear terahertz coherent excitation of vibrational modes of liquids," J. Chem. Phys 143, 234204 (2015).

4. Savolainen J, Ahmed S, and Hamm P, "Two-dimensional-Raman-terahertz spectroscopy of water," Proc. Natl. Acad. Sci 110(51), 20402-20407 (2013).

5. Finneran IA, Welsch R, Allodi MA, Miller III TF, and Blake GA, "Coherent two-dimensional terahertz-terahertz-Raman spectroscopy,” roc. Natl. Acad. Sci. U. S. A 113, 6857-6861 (2016).

6. Poulin PR and Nelson KA, "Irreversible organic crystalline chemistry monitored in real time," Scienc 313, 1756-1760 (2006).

7. Sakaibara H, Ikegaya Y, Katayama I, and Takeda J, "Single-shot time-frequency imaging spectroscopy using an echelon mirror,” Opt. Lett 37, 1118 (2012). [PubMed: 22446244]

8. Minami Y, Yamaki H, Katayama I, and Takeda J, "Broadband pump-probe imaging spectroscopy applicable to ultrafast single-shot events," Appl. Phys. Express 7, 022402 (2014).

9. Takeda J, Oba W, Minami Y, Saiki T, and Katayama I, "Ultrafast crystalline to-amorphous phase transition in Ge2Sb2Te5 chalcogenide alloy thin film using single-shot imaging spectroscopy," Appl. Phys. Let 104, 261903 (2014).

10. Kobayashi M, Minami Y, Johnson CL, Salmans PD, Ellsworth NR, Takeda J, Johnson JA, and Katayama I, "High-acquisition-rate single-shot pump-probe measurements using time-stretching method," Sci. Rep 6, 37614 (2016).

11. Kuribayashi T, Motoyama T, Arashida Y, Katayama I, and Takeda J, “Anharmonic phononpolariton dynamics in ferroelectric $\mathrm{LiNbO} 3$ studied with single-shot pump-probe imaging spectroscopy," J. Appl. Phys 123, 174103 (2018).

12. Minami Y, Hayashi Y, Takeda J, and Katayama I, "Single-shot measurement of a terahertz electricfield waveform using a reflective echelon mirror," Appl. Phys. Lett 103,51103 (2013).

13. Teo SM, Ofori-Okai BK, Werley CA, and Nelson KA, "Invited article: Single-shot THz detection techniques optimized for multidimensional THz spectroscopy,” Rev. Sci. Instrum 86, 051301 (2015).

14. Georges P, Brun A, Roger G, Le Saux G, and Salin F, "Single shot measurement of the optical Kerr effect kinetics," Appl. Opt 27, 777 (2009).

15. Zhang J, Liu S, Yi T, Wu X, Song Y, Zhang B, and Zhong Q, "Ultrafast single-shot measurement of optical Kerr effect based on supercontinuum pulse," Rev. Sci. Instrum 87, 43114(2016).

16. Hello P and Man CN, "Design of a low-loss off-axis beam expander," Appl. Opt 35,2534 (1996). [PubMed: 21085390]

17. Yan L, Wang X, Si J, He P, Chen F, Zou J, and Hou X, "Multi-frame observation of a single femtosecond laser pulse propagation using an echelon and optical polarigraphy technique," EEE Photonics Technol. Lett 25, 1879-1881 (2013).

18. Noe GT, Woods GL, Katayama I, Takeda J, Sullivan DM, Katsutani F, Zhang Q, Nojiri H, Hoffmann MC, Sekiguchi F, Horowitz JA, Allred JJ, and Kono J, "Single-shot terahertz timedomain spectroscopy in pulsed high magnetic fields," Opt. Express 24, 30328 (2016).

19. Khalil M, Golonzka O, Demirdöven N, Fecko CJ, and Tokmakoff A, "Polarization-selective femtosecond Raman spectroscopy of isotropic and anisotropic vibrational dynamics in liquids," Chem. Phys. Lett 321, 231-237 (2000).

20. Zhong Q and Fourkas JT, “Optical Kerr effect spectroscopy of simple liquids,” J. Phys. Chem. B 112, 15529-15539 (2008).

21. Degert J, Cornet M, Abraham E, and Freysz E, "Simple and distortion-free optical sampling of terahertz pulses via heterodyne detection schemes," Opt. Soc. Am. B 33,2045 (2016). 
22. Iliopoulos K, Potamianos D, Kakkava E, Aloukos P, Orfanos I, and Couris S, "Ultrafast third order nonlinearities of organic solvents," Opt. Express 23, 24171 (2015).

23. Heisler IA, Correia RRB, Buckup T, Cunha SLS, and da Silveira NP, "Time-resolved optical Kerreffect investigation on CS2/polystyrene mixtures,” J. Chem. Phys 123, 054509 (2005).

24. Fernández M, López JJ, Escribano RM, García-Ramos JV, Szalay V, de los Arcos T, FernándezLiencres MP, and Navarro A, "The force field of bromoform: A theoretical and experimental investigation,” J. Phys. Chem 100, 16058-16065 (2002).

25. Johnson TJ, Masiello T, and Sharpe SW, "The quantitative infrared and NIR spectrum of $\mathrm{CH}_{2} \mathrm{I}_{2}$ vapor: Vibrational assignments and potential for atmospheric monitoring," Amos. Chem. Phy 6, 2581-2591 (2006). 


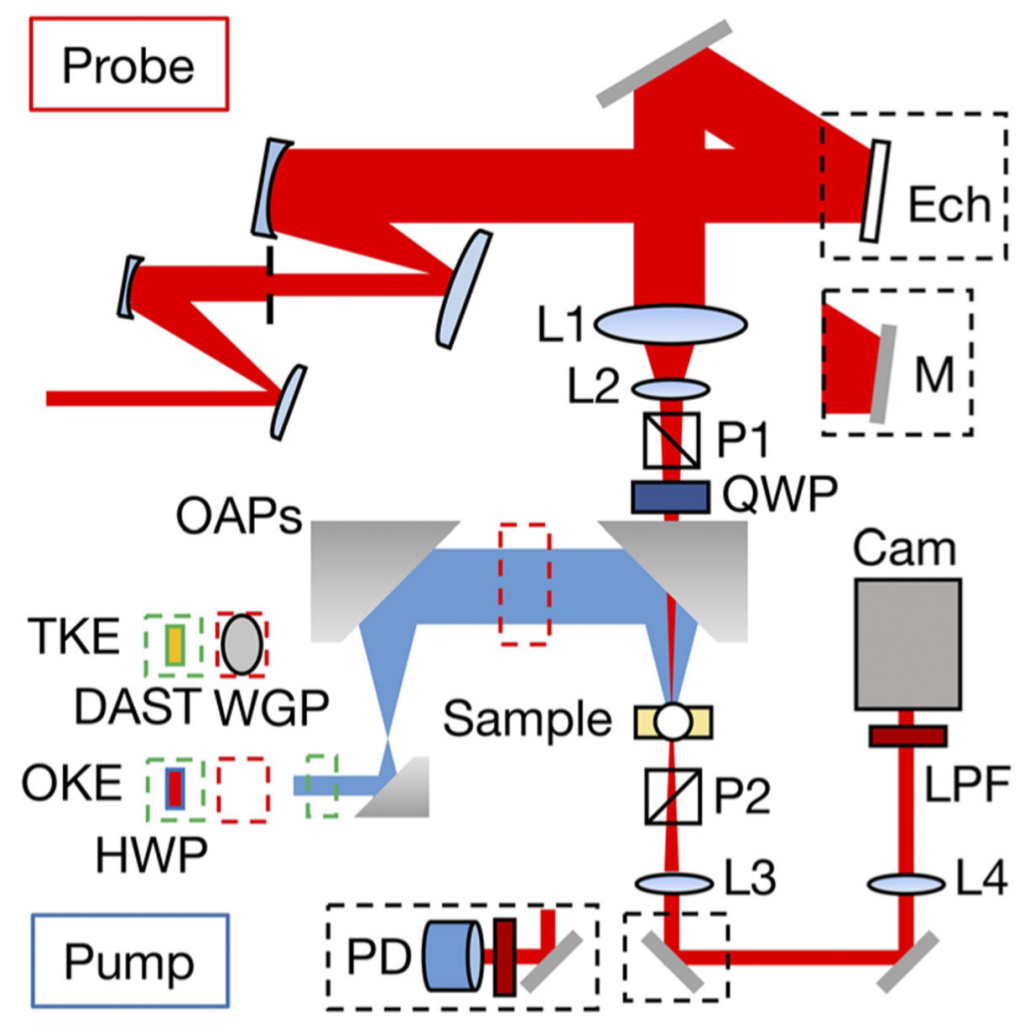

FIG. 1.

Diagram of the single shot experimental apparatus. The dashed black squares identify the echelon and post sample probe beam paths that were modified to perform reference stage scans. Dashed green and red squares indicate the optical components used for OKE and TKE measurements. DAST: THz organic crystal emitter, WGP: wire grid polarizer, HWP: half wave plate, Ech: reflective echelon, L1-L4: focusing and imaging lenses, P1, P2: polarizers, QWP: quarter wave plate, LPF: $750 \mathrm{~nm}$ long pass filter, Cam: high frame rate camera, PD: Si photo-diode, M: square mirror. 

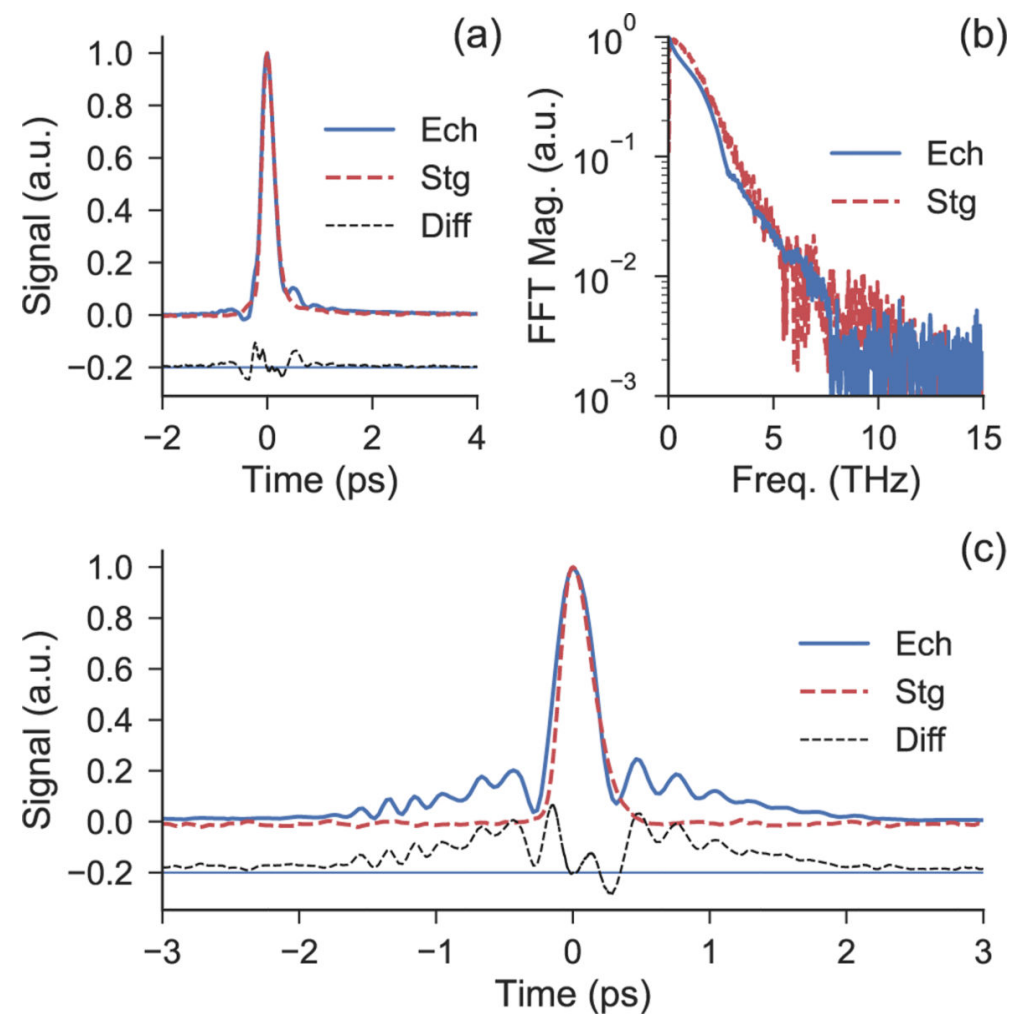

FIG. 2.

(a) Comparison of the OKE responses of DMSO measured using the stage scan (Stg) and echelon (Ech) techniques. Diff is the subtraction of the stage scan data from the echelon data, on the same scale and offset-0.2. (b) The FFT of the stage and echelon DMSO data demonstrate similar bandwidths and noise floors. (c) Same as in (a), but the OKE response of the Suprasil quartz cuvette. The diffraction features are clearly apparent around the main OKE response at $\mathrm{t}=0 \mathrm{ps}$. 
OKE
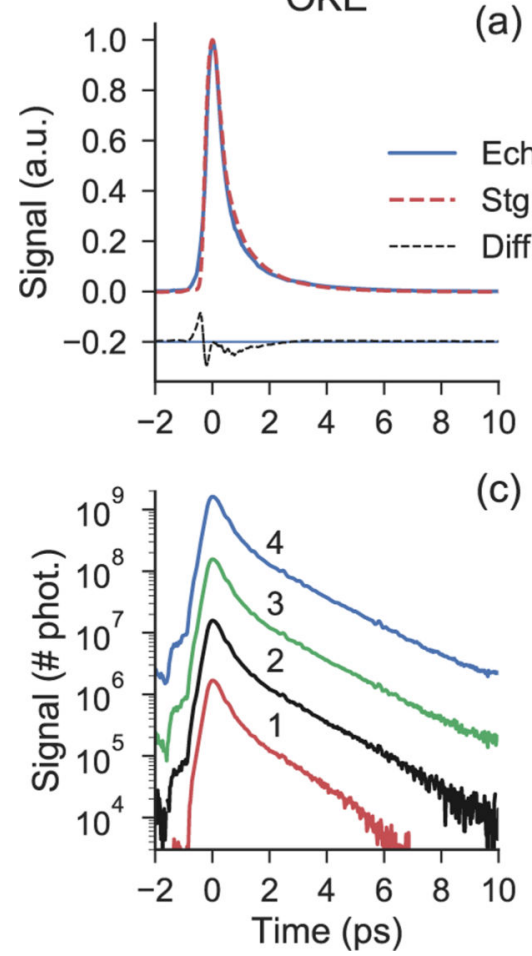

TKE

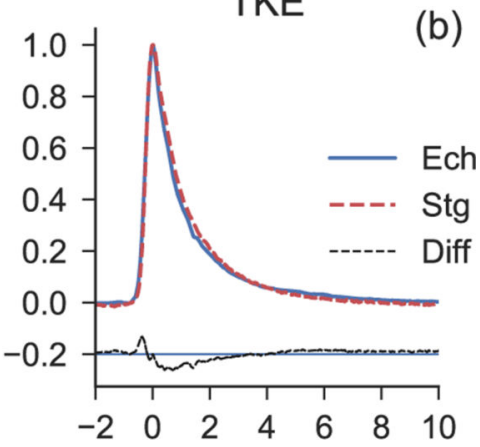

(d)

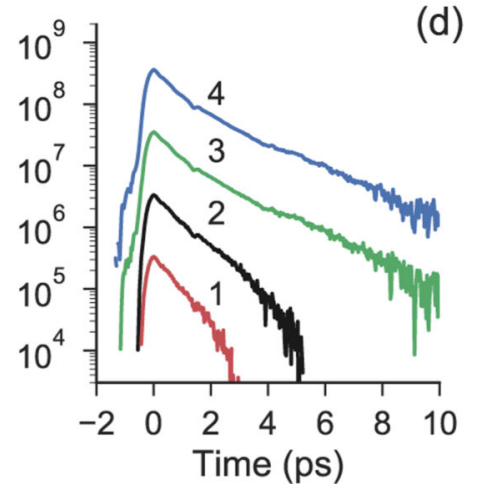

FIG. 3.

(a) Linear plots of the $\mathrm{CS}_{2}$ OKE signal recorded with the echelon (Ech) and stage scan (Stg) techniques as well as the difference between the two (Diff, -0.2). Good agreement between the two techniques is apparent out to $t=10 \mathrm{ps}$. (b) Similar to (a), but demonstrating the TKE response of $\mathrm{CS}_{2}$. (c) Evolution of the noise in the single shot $\mathrm{CS}_{2}$ OKE data across 4 orders of magnitude of sampling ( $\mathrm{N}=10^{x}$ shots, $\left.x=1,2,3,4\right)$. (d) The same analysis as in (c), but for the TKE response ofCS ${ }_{2}$. 

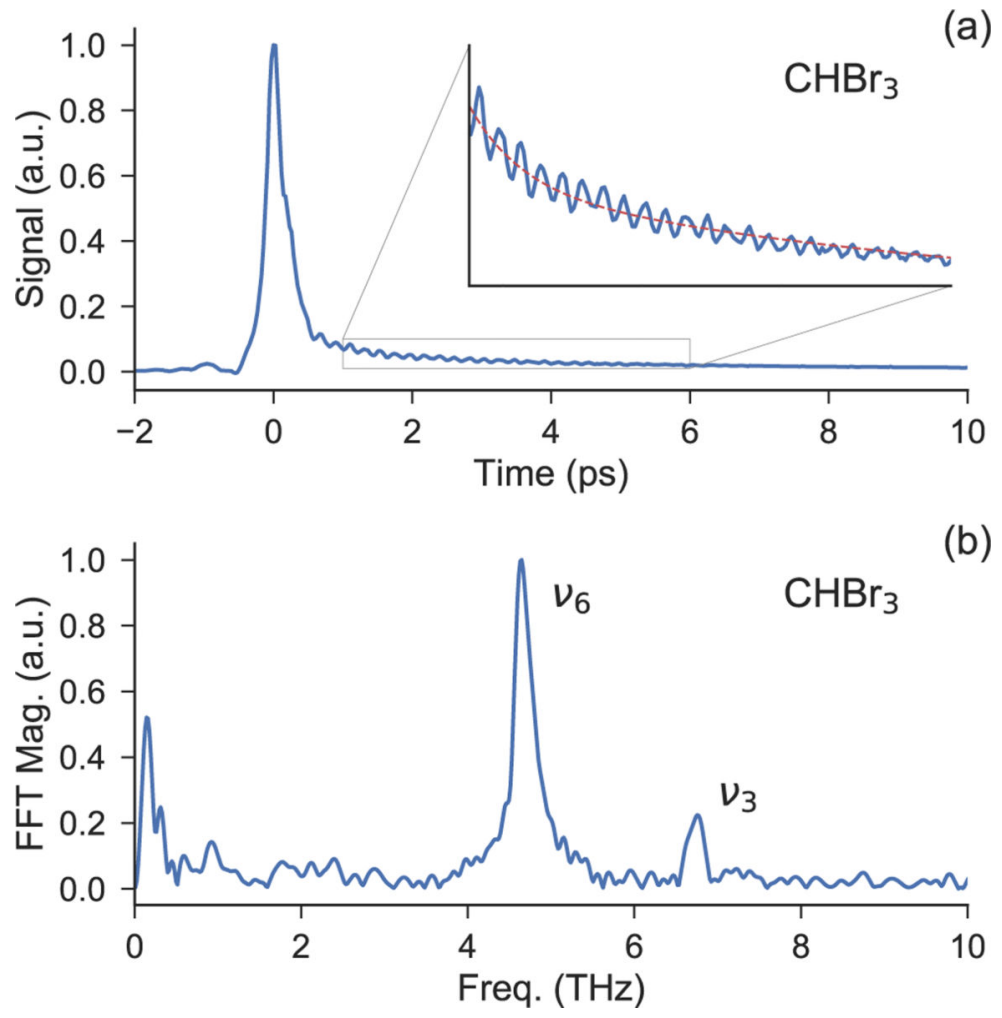

FIG. 4.

(a) Bromoform OKE response measured using the echelon approach ( $\mathrm{N}=10000$ shots). The oscillatory molecular coherences are shown in the inset, along with the orientational response fit. (b) The Fourier transform of the fit residual reveals the $\boldsymbol{v}_{\mathbf{6}}$ and $\boldsymbol{v}_{\mathbf{3}}$ Raman-active modes of bromoform. 

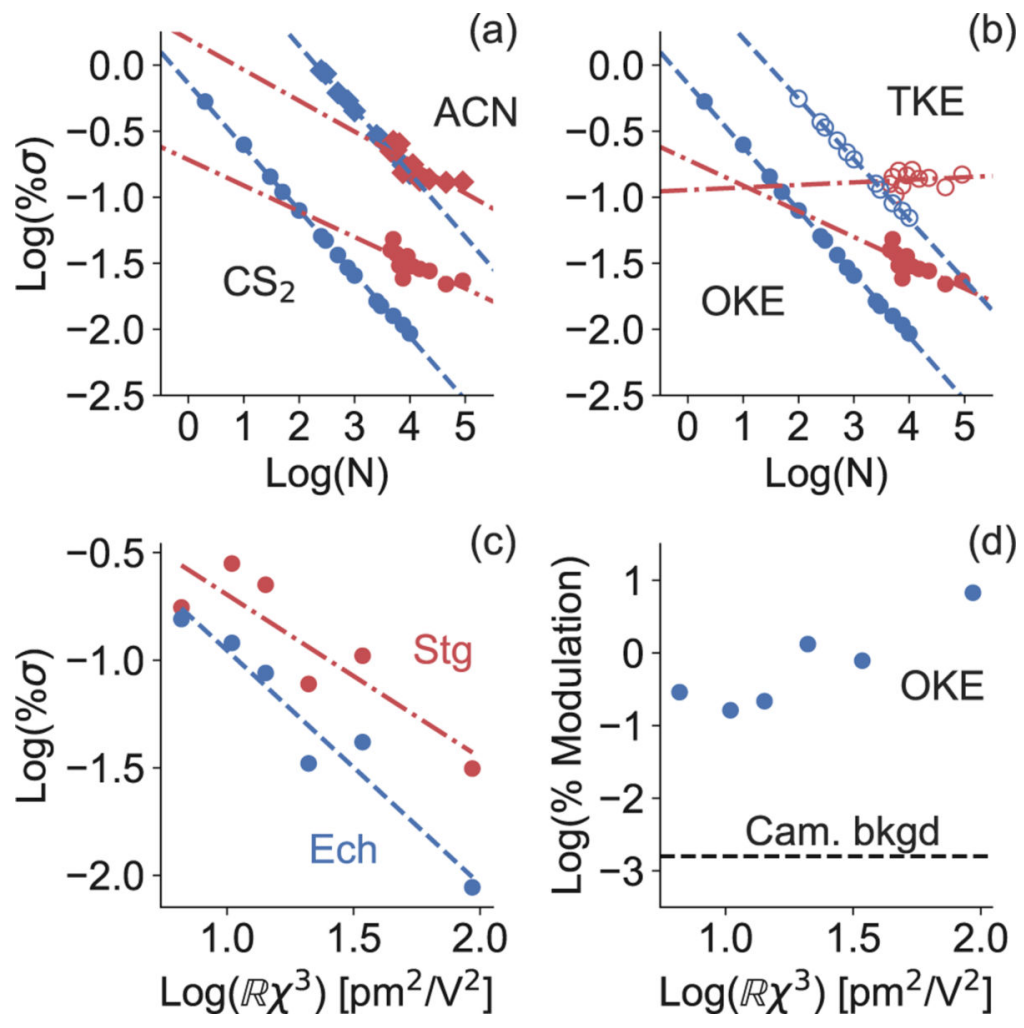

FIG. 5.

(a) The rms scaling of OKE signals from two solvents, acetonitrile (ACN, diamonds) and carbon disulfide $\left(\mathrm{CS}_{2}\right.$, circles), recorded using the echelon (blue) and stage scan (red) techniques. (b) Comparison between the rms noise performance of the two techniques in the OKE (filled markers) and TKE responses (empty markers) of $\mathrm{CS}_{2}$. While substantially weaker than OKE, TKE measurements obey similar trends in rms. (c) The OKE rms achieved after $\mathrm{N}=10000$ shots for a series of solvents, ranging from weakly to strongly Kerr active (plotted here as small to large $\chi^{3}$ constants). (d) The ratio of OKE signal photons to background photons (\% modulation) observed in the same range of solvents as in (c). The noise floor from random photon fluctuations with no pump present is shown as the dashed horizontal line. 